\section{What is a crystal?}

\author{
Ron Lifshitz* \\ Condensed Matter Physics 114-36, California Institute of Technology, \\ Pasadena, CA 91125, U.S.A.
}

Confusion is a word we have invented for an order which is not understood.

Henry Miller

Abstract. Almost 25 years have passed since Shechtman discovered quasicrystals, and 15 years since the Commission on Aperiodic Crystals of the International Union of Crystallography put forth a provisional definition of the term crystal to mean "any solid having an essentially discrete diffraction diagram." Have we learned enough about crystallinity in the last 25 years, or do we need more time to explore additional physical systems? There is much confusion and contradiction in the literature in using the term crystal. Are we ready now to propose a permanent definition for crystal to be used by all? I argue that time has come to put a sense of order in all the confusion.

\section{Periodicity or order?}

For almost two millennia crystallography was predominantly concerned with the external morphology of crystals. Crystallographers studied the naturally-occurring facets of crystals, which always intersect at precise and characteristic angles. It was only in the $17^{\text {th }}$ century that modern crystallography was born, thanks to the brilliant idea - attributed to such great scientists as Kepler and Hooke that crystal shapes were the result of internal order of "atomic" units.

In his study of tilings of the plane by polygons, Kepler [1] was quick to realize that very few regular polygons - namely the triangle, the square, and the hexagon can tile the plane without introducing overlaps or leaving holes. Yet, this observation did not deter him from constructing a well-ordered tiling of the plane, consisting of pentagons and decagons that requires some of the decagons to overlap, and leaves holes in the form of 5-fold stars - a tiling that was to be rediscovered by Penrose more than 350 years later [2, 3]. Without realizing it, Kepler had discovered some of the basic properties of aperiodic order. In his words:

"If you really wish to continue the pattern, certain irregularities must be admitted, two decagons must be combined, two sides being removed from each of

* On leave from Tel Aviv University. E-mail: ronlif@tau.ac.il them. As the pattern is continued outwards five-cornered forms appear repeatedly: ... So as it progresses this five-cornered pattern continually introduces something new. The structure is very elaborate and intricate."

Unfortunately, by the end of the $18^{\text {th }}$ century, when Haüy began formulating the mathematical theory of crystallography [4], Kepler's insightful drawings of aperiodic tilings with decagonal symmetry were long forgotten. Mathematical crystallography was founded upon the premise that the internal order of crystals was necessarily achieved through a periodic filling of space. Thus, crystallography treated order and periodicity synonymously, using either property interchangeably to define the notion of a crystal. The periodic nature of crystals was "confirmed" with the discovery of x-ray crystallography and numerous other experimental techniques throughout the $20^{\text {th }}$ century. Being more "elaborate and intricate" and less commonly found in Nature, aperiodic crystals were completely overlooked. Periodicity became the underlying paradigm, not only for crystallography itself, but also for other disciplines such as materials science and condensed matter physics, whose most basic tools, like the Brillouin zone, rely on its existence.

This historical oversight was corrected with Shechtman's dramatic discovery of quasicrystals in 1982 [5] - a discovery that sparked a bona fide Kuhnian scientific revolution [6, 7]. In the famous Hargittai interviews [8], with all the scientists involved in the initial study of quasicrystals, Mackay is quoted as saying that

"It's a discovery of a material which breaks the laws that were artificially constructed. They were not laws of nature; they were laws of the human classificatory system."

Nature had found a way of achieving order without periodicity, and Shechtman was careful enough not to dismiss it as an experimental artifact, as many must have done before him. He confronted a skeptical scientific community that was unwilling to relinquish its most basic paradigm that order stems from periodicity. In an article, suggesting a description of Shechtman's icosahedral quasicrystal as multiple twinning of periodic cubic crystals - a description that was later shown to be incorrect [9] Pauling [10] concluded by saying that

"Crystallographers can now cease to worry that the validity of one of the accepted bases of their science has been questioned."

Today, thousands of diffraction diagrams later, compounded by high-quality experimental data - such as images from high-resolution transmission electron microscopes and atomic-resolution scanning tunneling microscopes - the existence of order without periodicity has been unequivocally established. Not only has the periodicity paradigm been questioned, as Pauling worried, it has 
been completely invalidated. It is time now, as Ben-Abraham [11] argues in this volume, to make a clear statement associating the notion of a crystal with its more fundamental and underlying property of order, rather than the property of periodicity, which is only one way of achieving order (even if it is the more common way in which Nature chooses to do so). According to this, crystals that are periodic should explicitly be called periodic crystals, all others should be called aperiodic crystals.

Some may object by saying that there are no right or wrong definitions, and that a definition is merely a matter of mutual agreement. They would further claim that the notion of a crystal should strictly be associated with periodicity, because over a century of literature in mathematical crystallography contains statements and proofs about crystals that are, in fact, true only for periodic crystals. A change of the definition would cause great confusion when reading this literature. This type of argument is typical of the paradigmshifting stage we are currently undergoing in this scientific revolution [12]. Nevertheless, the discovery of quasicrystals demands such a paradigm shift and along with it a new definition of the notion of a crystal. One simply needs to be aware, whenever reading old literature, that the term crystal may be used in its old restricted meaning.

\section{But, what is order?}

In 1992, the International Union of Crystallography, through its Commission on Aperiodic Crystals [13], published a provisional definition of the term crystal that abolishes periodicity, but does not go as far as naming order as its replacement. The commission was not ready to give precise microscopic descriptions of all the ways in which order can be achieved. Clearly, periodicity is one way of achieving order, quasiperiodicity as in the PenroseKepler tiling is another, but can we be certain that there are no other ways that are yet to be discovered? The Commission opted to shift the definition from a microscopic description of the crystal to a property of the data collected in a diffraction experiment. It decided on a temporary working-definition whereby a crystal is

\section{"any solid having an essentially discrete diffraction} diagram."

The new definition was left sufficiently vague so as not to impose unnecessary constraints until a better understanding of crystallinity emerges.

The 1992 definition is consistent with the notion of long-range order - one of the basic notions of condensedmatter physics [14, 15] - dating back to ideas of Landau in which the symmetry-breaking transition from a disordered (high-symmetry) phase to an ordered (low-symmetry) phase is quantified by the appearance of a non-zero order parameter. Stated in plain words, long-range order - or in the context of our current discussion, longrange positional order - is a measure of the correlations between the positions of atoms in distant regions of the material. It measures the extent of our ability to describe the positions of atoms in far away regions of space, based on our knowledge of their positions nearby.
How is this related to the requirement of having an essentially discrete diffraction diagram? If $\rho(\boldsymbol{r})$ is a density function, describing a certain material, then the function that measures correlations between two points, separated in space by a vector $\boldsymbol{R}$ - the two-point autocorrelation function-is known in crystallography as the Patterson function,

$$
P(\boldsymbol{R})=\lim _{V \rightarrow \infty} \frac{1}{V} \int \mathrm{d} \boldsymbol{r} \rho(\boldsymbol{r}) \rho(\boldsymbol{r}-\boldsymbol{R}) .
$$

Its Fourier transform is exactly the intensity $I(\boldsymbol{k})=|\rho(\boldsymbol{k})|^{2}$ that is measured in most diffraction experiments. It was realized long ago (e.g. Ref. [16]), that the order parameter that signals a transition from a disordered liquid to an ordered solid, indicating the emergence of non-trivial correlations as measured by the Patterson function (1), appears in the form of delta functions, or Bragg peaks, at non-zero wave vectors in the diffraction diagram. Thus, the observation of essentially discrete points in the diffraction diagram is the indication of having long-range positional order, and therefore of crystallinity. From the point of view of condensed-matter physics, long-range positional order is characterized by having Bragg peaks in the Fourier transform of the density function $\rho(\boldsymbol{r})$. In the language of spectral theory, the diffraction spectrum of $\rho(\boldsymbol{r})$ must contain a pure-point component, for $\rho(\boldsymbol{r})$ to be considered crystalline.

\section{Periodic, quasiperiodic, and almost-periodic crystals}

Following H. Bohr's theory of almost periodic functions [17], solids whose density functions $\rho(\boldsymbol{r})$ may be expanded as a superposition of a countable number of plane waves

$$
\rho(\boldsymbol{r})=\sum_{\boldsymbol{k} \in L} \rho(\boldsymbol{k}) e^{i \boldsymbol{k} \cdot \boldsymbol{r}}
$$

are to be called almost periodic crystals. In particular, if taking integral linear combinations of a finite number $D$ of wave vectors in this expansion can span all the rest, then the crystal is to be called quasiperiodic. The diffraction pattern of a quasiperiodic crystal, therefore, contains Bragg peaks, each of which can be indexed by $D$ integers. If $D$ is the smallest number of wave vectors that can span the whole set $L$ using integral linear combinations, then $D$ is called the rank, or the indexing dimension of the crystal. Periodic crystals form a special subset of all quasiperiodic crystals, whose rank $D$ is equal to the actual physical dimension $d$ (the number of components in the vectors $\boldsymbol{r}$ and $\boldsymbol{k}$ ).

For periodic crystals the set of Bragg peaks is truly discrete, because the set of wave vectors $\boldsymbol{k}$ in their Fourier expansion (2) is discrete. For quasiperiodic crystals whose rank $D$ is greater than the physical dimension $d$, the set $L$ of wave vectors in the expansion (2) is dense - there are $\boldsymbol{k}^{\prime}$ 's in $L$ that cannot be surrounded by a finite $d$-dimensional ball that contains no other $\boldsymbol{k}$ 's. Nevertheless, in actual experiments, where the total integrated diffraction intensity is finite, Bragg peaks are not observed at wave vectors $\boldsymbol{k}$ for which the intensity $|\rho(\boldsymbol{k})|^{2}$ is below a certain threshold. In this sense the observed diffraction pattern is essentially dis- 
crete, abiding by the 1992 definition, even though the set $L$ is not discrete. As the resolution of the diffraction experiment is improved, additional peaks will appear in the diffraction diagram. If the crystal is periodic the added peaks will appear further away from the origin. If the crystal is quasiperiodic, new peaks can appear in between already-existing ones, yet all the new peaks will still be indexable by $D$ integers. If the crystal is almost periodic, new vectors may be required in order to generate the additional peaks, thus the rank $D$ will seem to grow as the resolution is improved.

It should be noted that all experimentally observed crystals to date are quasiperiodic. The term quasicrystal, which was first introduced by Levine and Steinhardt [18], is simply short for quasiperiodic crystal, but is used to refer to those quasiperiodic crystals that are strictly aperiodic (i.e. with $D>d$ ). Some authors require crystals to possess so-called "forbidden symmetries" in order to be regarded as quasicrystals. I have explained elsewhere [19] why such a requirement is inappropriate.

One should also recall that certain classes of quasiperiodic crystals were known long before Shechtman's discovery. These are the so-called incommensurately-modulated crystals and incommensurate composite crystals, (or intergrowth compounds). These crystals did not pose any serious challenge to the periodicity paradigm because they could all be viewed as periodic structures that had been slightly modified. Order was still obtained through periodicity, leaving the paradigm intact.

Almost periodic crystals have not (yet) been found in Nature, but can be constructed artificially. A possible way of constructing such crystals is to consider the limit of an infinite sequence of periodic or quasiperiodic structures. If chosen properly, such a limit can yield so-called limit-periodic or limit-quasiperiodic structures, whose spectrum was shown to consist of Bragg peaks, even though their rank is infinite [20].

\section{What else is crystalline?}

The discovery of quasicrystals and the possibility for aperiodic order has made a great impact on mathematics [2124], as summarized by Lagarias [25]. Much effort has been invested in studying the characteristics of order, as well as in the development of diffraction theory [26, 27], once it was realized that periodicity was not a necessary condition for order and for the appearance of Bragg peaks in the diffraction spectrum.

One interesting line of study [25] is an attempt to characterize the local mathematical properties of periodic crystals and quasicrystals that are responsible for the emergence of long-range order. For periodic crystals it is wellunderstood that any of a number of local properties is sufficient for ensuring periodicity, and thus order. These notions can be generalized to characterize the local properties of quasicrystals, yet it is still unclear whether any of these notions can serve as a sufficient condition for ensuring quasiperiodicity, or more generally, long-range order.

Rather than trying to characterize local structural properties that ensure Bragg peaks in the diffraction, a number of authors have considered the diffraction properties of structural models that are constructed by particular methods. Could there be a constructive procedure that perfectly encompasses all structures that possess long-range order? The answer to this question is still unknown, although a number of unintuitive and even surprising results have already been obtained.

It is well-understood that structure models that are constructed by the cut-and-project technique from finite dimensional spaces [21] are all quasiperiodic, and therefore have a Bragg spectrum and possess long-range order. The same holds for structures that are generated using the dual grid method and its various generalizations [28, 29]. These construction methods are therefore too limited to encompass all ordered structures. On the other hand, it turns out that other approaches that are commonly used to generate quasicrystal models are too general, often producing structures that do not possess Bragg peaks in their diffraction spectrum, and therefore are not crystalline.

A typical example of a construction method that is too general to be used to define crystallinity is that of substitution rules-either of letter sequences in one dimension, or of tiles in two or three dimensions. The famous Fibonacci sequence, produced by the substitution rules $L \rightarrow L S$ and $S \rightarrow L$, produces a rank-2 quasicrystal. What may seem as a minor change to these substitution rules may generate sequences that do not have a Bragg spectrum. Models based on such sequences do not possess long-range order and are therefore not crystals, even though they are constructed using the same deterministic procedure that is used to generate many quasicrystals. It has been understood for some time [30] that what determines whether a structure, generated by substitution, has long-range order is an algebraic property of the matrix, which describes the substitution. The generated structure contains Bragg peaks in its Fourier spectrum only if the largest eigenvalue of the substitution matrix is a so-called Pisot-Vijayaraghavan number.

The fact that there are no correlations between the letters of a sequence that is constructed by a deterministic procedure may seem counter-intuitive, but readers who are familiar with the notion of deterministic chaos may not be so alarmed. Ben-Abraham [11] has suggested considering all structures that are generated by deterministic procedures as crystals. Along the same lines, Mackay [31] has argued that

"a crystal is a structure the description of which is
much smaller than the structure itself."

I object to such definitions that confuse the notion of order with determinism. As counter-intuitive as it may seem, deterministically-generated structures might be disordered. If this were not the case it would not have been possible to design computer algorithms that generate random numbers. Random number generators use deterministic procedures to generate uncorrelated numbers that look as if they were obtained by the random flipping of a coin. When considering the actual structures, deterministic disorder is practically the same as random disorder, and should not be associated with crystallinity. Not every deterministic structure that is simple to generate is necessarily a crystal.

Baake and several co-workers [32-36] are currently performing a systematic study whose purpose is to charac- 
terize which distributions of matter diffract to produce a pure point component in their spectrum, and thus can qualify as possessing long-range order. In some cases it is even difficult to determine whether the Fourier transform of a structure exists, in the sense that it has a unique infinite volume limit. When it is clear that the Fourier transform exists it may still be difficult to rigorously know whether it contains Bragg peaks. This is a difficult study which might take a long time to complete, yet it is an important study that may eventually provide a proper characterization of long-range order.

\section{The gray zone}

There are many structures that are partially-ordered-either by design, or due to "practical" circumstances such as thermal fluctuations, or the existence of defects and grain boundaries. In some of these cases it might be difficult to decide at what point the structure ceases to be crystalline. Often, details regarding the shape of Bragg peaks offer a convenient way of doing so. Melting of a crystal due to thermal fluctuations, for example, may be formulated in terms of a real-space condition such as the Lindemann criterion, or as a condition on the reduction of Bragg-peak intensities due to the Debye-Waller factor. Finite-size issues can be formulated as a criterion of minimum size in real space, or as a condition on the width of Bragg peaks or their scaling with the number of atoms. In some of these cases it is a matter of taste to decide what constitutes a crystal, and one may need to rely on common sense. Questions such as "how many atoms are needed to form a crystal?" or "when is an atomic cluster sufficiently large to be considered a nanocrystal?" should not be of any real concern. On the other hand, questions of short-range versus long-range order in a given homogeneous material should be considered with care.

More interesting are structures that by design possess both long-range order and some degree of disorder. An important example is that of random-tiling models of quasicrystals [37, 34]. These models are believed to have an underlying Bragg spectrum, indicative of quasiperiodic long-range order, superimposed with a diffuse background stemming from phason disorder, which is inherent to the randomness of the models. In this case, the underlying Bragg spectrum is often viewed as being the essential component, and therefore the structure is considered to be crystalline.

An opposite example is the one-dimensional ThueMorse sequence, generated from two letters by the substitution rule $L \rightarrow L S$ and $S \rightarrow S L$. If represented as a sequence of short $(S)$ and long $(L)$ segments with atoms at the joining points, then $1 / 2$ of the atoms form a periodic lattice with a unit cell of length $S+L$. The remaining atoms divide the unit cell into an $L$ followed by an $S$, or vice versa, in a completely disordered fashion. In this case, many authors tend to view the underlying periodic backbone as an unimportant artifact, and the structure itself as being disordered. In fact, one can carefully choose to represent the Thue-Morse sequence in a non-generic way in which all of the Bragg peaks become extinguished, leaving only the diffuse part of the diffraction spectrum [30]. If viewed as being essentially disordered, the Thue-Morse sequence should not be regarded as a crystal, although one should always remember that it does contain an underlying periodic lattice.

\section{So, what is a crystal?}

Having said all this, I would like to conclude with a definition:

1. A crystal is a solid that has long-range positional order.

2. Long-range positional order can be inferred from the existence of Bragg peaks in the Fourier spectrum of the solid.

It is common to add a descriptive prefix to the term crystal to refer to soft materials, metamaterials, or any other form of matter that is crystalline but is not an atomic solid. Examples are photonic crystals and liquid or soft crystals - more appropriately called crystalline liquids [38] to distinguish them from the more common liquid crystals that have long-range orientational rather than positional order. These should now be understood in the context of the new definition of crystal to include periodic soft and photonic crystals as well as soft quasicrystals [39, 40] and photonic quasicrystals [41-44] that are not periodic.

I would happily welcome a revision of the second part of the definition once a more complete characterization of order is established. In the meantime, one must follow the only reliable characterization in terms of the Fourier spectrum. I would be even happier to welcome a second scientific revolution in materials science that would require yet another paradigm shift and a completely new definition. Until that happens, I suggest sticking to order.

Acknowledgments: I thank S.I. Ben-Abraham for insisting that we reconsider now the definition of crystal, rather than wait for another 10 or 15 years. I also thank the Editor of Zeit. Krist., Walter Steurer, for suggesting to me that I put my thoughts on this matter into writing. Finally, I thank David Mermin for his thoughtful comments on this manuscript.

My research in crystallography is currently supported by the Israel Science Foundation through grant number 684/06.

\section{References}

[1] Kepler, J.: Harmonices Mundi, (Frankfurt, 1619) Book II. English translation by E. J. Aiton, A. M. Duncan, and J. V. Field: The harmony of the world. Am. Philos. Society, (1997).

[2] Penrose, R.: The role of aesthetics in pure and applied mathematical research. Bull. Inst. Math. Appl. 10 (1974) 266.

[3] Gardner, M.: Sci. Am. 236 (1977) 110.

[4] Haüy, R.-J.: Essai d'une Théorie sur la Structure des Crystaux. Gogué \& Née, Paris, (1784).

[5] Shechtman, D.; Blech, I.; Gratias, D.; Cahn, J. W.: Metallic phase with long-ranged orientational order and no translational symmetry. Phys. Rev. Lett. 53 (1984) 1951.

[6] Cahn, J. W.: Epilogue. In: Proceedings of the 5th International Conference on Quasicrystals (Ed. C. Janot and R. Mosseri). World Scientific, Singapore, (1996) 807.

[7] Lifshitz, R.: The rebirth of crystallography. Z. Kristallogr. 217 (2002) 432

[8] Hargittai, I.: Quasicrystal discovery: A personal account. Chem. Intell. 3(4) (1997) 25. 
[9] See, for example: Bancel, P. A.; Heiney P. A.; Horn, P. M.; Steinhardt, P. J.: Comment on a Paper by Linus Pauling. Proc. Natl. Acad. Sci. USA 86 (1989) 8600.

[10] Pauling, L.: Apparent icosahedral symmetry is due to directed multiple twinning of cubic crystals. Nature 317 (1985) 512.

[11] Ben-Abraham, S. I.: What is a crystal? Z. Kristallogr. 222 (2007) 310 .

[12] Kuhn, T. S.: The Structure of Scientific Revolutions. University of Chicago Press, (1962).

[13] International Union of Crystallography: Report of the executive committee for 1991. Acta Cryst. A48 (1992) 922; see p. 928.

[14] Anderson, P. W.: Basic notions of condensed matter physics. Addison Wesley, Reading MA, (1997), Chap. 2.

[15] Sethna, J. P.: Entropy, order parameters, and complexity. Clarendon Press, Oxford, (2006), Chap. 9.

[16] Mermin, N. D.: Crystalline order in two dimensions. Phys. Rev. 176 (1968) 250.

[17] Bohr, H.: Almost periodic functions. Chelsea, New York, (1947).

[18] Levine, D.; Steinhardt, P. J.: Quasicrystals I: Definition and structure. Phys. Rev. B34 (1986) 596.

[19] Lifshitz, R.: Quasicrystals: A matter of definition. Foundations of Physics 33 (2003) 1703.

[20] Baake, M.; Moody, R. V.; Schlottmann, M.: Limit-(quasi) periodic point sets as quasicrystals with p-adic internal spaces. J. Phys. A: Math. Gen. 31 (1998) 5755.

[21] Senechal, M.: Quasicrystals and Geometry. Cambridge Univ. press, Cambridge, (1996).

[22] Moody, R. V. (Ed.): The Mathematics of Long-Range Aperiodic Order. Kluwer, Dordrecht, (1997).

[23] Patera, J. (Ed.): Quasicrystals and discrete geometry. American Mathematical Society, Providence, (1998).

[24] Baake, M.; Moody, R. V. (Eds.): Directions in Mathematical Quasicrystals. American Mathematical Society, Providence, (2000).

[25] Lagarias, J. C.: The impact of aperiodic order on mathematics. Materials Science and Engineering 294-296 (2000) 186.

[26] Hof, A.: Diffraction by aperiodic structures. In Ref. [22], p. 239.

[27] Lagarias, J. C.: Mathematical quasicrystals and the problem of diffraction. In Ref. [24], p. 61.

[28] de Bruijn, N. G.: Proc. K. Ned. Akad. Wet., Ser. A: Math. Sci. 84 (1981) 39.
[29] Gähler, F.; Rhyner, J.: Equivalence of the generalised grid and projection methods for the construction of quasiperiodic tilings. J. Phys. A19 (1986) 267.

[30] Gähler, F.; Klitzing, R.: The diffraction pattern of self-similar tilings. In Ref. [22], p. 141

[31] Mackay, A. L.: Generalized crystallography. Structural Chemistry 13 (2002) 215

[32] Baake, M.; Moody, R. V.: Diffractive point sets with entropy. J. Phys. A31 (1998) 9023.

[33] Baake, M.; Moody, R. V.; Pleasants, P. A. B.: Diffraction from visible lattice points and kth power free integers. Disc. Math. 221 (2000) 3.

[34] Baake, M.; Höffe, M.: Diffraction of random tilings: Some rigorous results. J. Stat. Phys. 99 (2000) 219.

[35] Höffe, M.; Baake, M.: Surprises in Diffuse Scattering. Z. Kristallogr. 215 (2000) 441.

[36] Baake, M.; Moody, R. V.; Richard, C.; Sing, B.: Which distribution of matter diffracts? In: Quasicrystals: Structure and Physical Properties. (Ed. H.-R. Trebin) Wiley-VCH, Berlin, (2003) 188.

[37] Henley, C. L.: Random tiling models. In: Quasicrystals - The State of the Art. (D. P. DiVincenzo and P. J. Steinhardt, Eds.) World Scientific, Singapore, (1991), p. 429.

[38] Wright, D. C.; Mermin, N. D.: Crystalline liquids: the blue phases. Rev. Mod. Phys. 61 (1989) 385.

[39] Zeng, X.; Ungar, G., Liu, Y.; Percec, V.; Dulcey, A. E.; Hobbs, J. K.: Supramolecular dendritic liquid quasicrystals. Nature $\mathbf{4 2 8}$ (2004) 157.

[40] Lifshitz, R.; Diamant, H.: Soft quasicrystals - Why are they stable? To appear in Phil. Mag. (preprint cond-mat/0611115).

[41] Jin, C.; Cheng, B.; Man, B.; Li, Z.; Zhang, D.; Ban, S.; Sun, B.: Band gap and wave guiding effect in a quasiperiodic photonic crystal. Appl. Phys. Lett. 75 (1999) 1848.

[42] Zoorob, M. E.; Charlton, M. D. B.; Parker, G. J.; Baumberg, J. J.; Netti, M. C.: Complete photonic bandgaps in 12-fold symmetric quasicrystals. Nature 404 (2000) 740.

[43] Lifshitz, R.; Arie, A.; Bahabad, A.: Photonic quasicrystals for nonlinear optical frequency conversion. Phys. Rev. Lett. 95 (2005) 133901.

[44] Freedman, B.; Bartal, G.; Segev, M.; Lifshitz, R.; Christodoulides, D. N.; Fleischer, J. W.: Wave and defect dynamics in nonlinear photonic quasicrystals. Nature 440 (2006) 1166. 